\title{
La chloroquine peut être toxique pour le muscle, il ne faut pas l'oublier !
}

\section{J. Andoni Urtizberea}

Résumé

Dans cet article [1], des neurologues américains de Miami rapportent l'observation d'une femme de race blanche de 65 ans, sans antécédents médicaux, présentant un déficit moteur progressif survenu de manière insidieuse au niveau des membres inférieurs et supérieurs. Cette faiblesse avait été précédée par un épisode de douleurs thoraciques de quelques jours, pour lequel le diagnostic de péricardite d'origine possiblement lupique a été retenu. Dans cette hypothèse, un traitement par hydroxychloroquine avait été entrepris à raison de $200 \mathrm{mg}$ journaliers. Il n'y avait toutefois ni signes cutanés ni signes articulaires et le reste du bilan biologique était sans particularité. La patiente prenait par ailleurs du losartan pour traiter une hypertension artérielle ancienne mais n'avait jamais été exposée aux statines. L'examen neurologique montrait un déficit moteur relativement peu sélectif, à prédominance proximale, sans troubles sensitifs, ni myotonie, ni signes respiratoires associés. L'EMG était myogène et les CPK aux alentours de 1200 unités par litre. La constatation d'un déficit musculaire progressif dans les semaines ayant suivi la prise d'hydroxychloroquine a fait d'abord suspecter une pathologie neuromusculaire secondaire d'origine iatrogène. Ce d'autant que la biopsie musculaire, réalisée au niveau d'un quadriceps, avait révélé la présence d'une myopathie vacuolaire typique. La mise en évidence, dans les vacuoles, d'un matériel glycogénique positif en PAS a toutefois fait évoquer le diagnostic alternatif de forme tardive de maladie de Pompe. Dans ce contexte, une enzymothérapie substitutive a été débutée, sans preuves biologiques formelles semble-t-il, et interrompue au bout de six mois en l'absence de réponse clinique franche. Le bilan refait en neurologie à Miami a permis d'écarter le diagnostic de maladie de Pompe devant la normalité des tests enzymatiques et l'absence de mutations pathogènes du gène GAA. C'est finalement l'arrêt de l'hydrochloroquine qui a permis, au bout de quelques semaines, une nette stabilisation du déficit moteur et une normalisation du taux sérique de $\mathrm{CPK}$, confirmant ainsi le diagnostic de myopathie toxique.

\section{Commentaire}

Cette observation illustre bien les difficultés rencontrées dans l'exploration de troubles musculaires à un âge avancé, période de la vie où les étiologies, autoimmunes, génétiques ou iatrogènes, sont souvent intriquées. Même s'il est bon d'évoquer systématiquement la possibilité d'une forme tardive de maladie de Pompe, il faut se garder de surdiagnostiquer cette affection et pire encore, de débuter un traitement lourd comme l'enzymothérapie en l'absence de preuves biologiques formelles. C'est l'occasion aussi de rappeler, en ces temps de pandémie Covid-19, l'existence de véritables myopathies toxiques liées à l'hydroxychloroquine. Ces dernières ont été rapportées pour la première fois dans les années 1970 suite à la découverte des bienfaits du Plaquénil® dans les maladies de système, et plus particulièrement, la polyarthrite rhumatoïde et le lupus aigu disséminé. L'hydroxychloroquine, tout comme la chloroquine, agissent au niveau du lysosome et bloquent plusieurs processus enzymatiques. Cette toxicité peut s'exercer au niveau du muscle squelettique, du myocarde, mais aussi du nerf, à des doses journalières comprises entre 200 et $400 \mathrm{mg}$. La survenue d'une myopathie, d'une cardiomyopathie ou d'une neuromyopathie reste toutefois rare et aléatoire dans le temps (le délai d'apparition varie de plusieurs semaines à plusieurs mois). Le tableau clinique est, en dehors du déficit musculaire, relativement pauvre. Les lésions observées sur la biopsie musculaire sont celles d'une myopathie vacuolaire mais peuvent parfois mimer une glycogénose en tout point.

Le diagnostic de myopathie à la chloroquine ou à l'hydroxychloroquine reste toutefois un diagnostic d'exclusion, d'où la nécessité d'un bilan étiologique large, notamment génétique (panel de gènes de myopathie des ceintures), biochimiques (dosages enzymatiques) et immunologique (dosages d'auto-anticorps spécifiques) lorsqu'une symptomatologie myopathique ou neuromyopathique survient chez une personne d'âge mûr.

Chloroquine can be toxic to the muscle, let's not forget it!

\section{LIENS D'INTÉRÊT}

L'auteur déclare n'avoir aucun lien d'intérêt concernant les données publiées dans cet article. 
RÉFÉRENCE

1. Shukla S, Gultekin SH, Saporta M. Pearls \& Oy-sters: hydroxychloroquine-induced toxic myopathy mimics Pompe disease. Critical role of genetic test. Neurology 2019; 92 : e742-5.

POUR ALLER PLUS LOIN SUR LE SUJET

1. Amato AA, Russell JA. Neuromuscular disorders, $2^{\text {nd }}$ ed. New York : McGraw-Hill, 2015.

2. Wolfe GI, Baker NS, Haller RG, Burns DK, Barohn RJ. McArdle's disease presenting with asymmetric, late-onset arm weakness. Muscle Nerve $2000 ; 23: 641-5$

3. Casado E, Gratacos J, Tolosa C, et al. Antimalarial myopathy: an underdiagnosed complication? Prospective longitudinal study of 119 patients. Ann Rheum Dis $2006 ; 65: 385-90$.

4. Avtna-Zubieta JA, Johnson ES, Suarez-Almazorj ME, Russell AS. Incidence of myopathy in patients treated with antimalarials: a report of 3 cases and a review of literature. Br J Rheumatol 1995; 34 : 166-70.

5. Estes ML, Ewing-Wilson D, Chou SM, et al. Chloroquine neuromyotoxicity, clinical and pathologic perspective. Am J Med 1987 ; $82: 447$.

6. Abdel-Hamid H, Oddis CV, Lacomis D. Severe hydroxychloroquine myopathy. Muscle Nerve 2008 ; 38 : 1206-10.

7. Stein M, Bell MJ, Ang LC. Hydroxychloroquine neuromyotoxicity. J Rheumatol $2000 ; 27: 2927$.

8. Richards AJ. Hydroxychloroquine myopathy. J Rheumatol 1998; 25 : 1642-3.

9. Siddiqui AK, Huberfeld SI, Weidenheim KM, Einberg KR, Efferen LS. Hydroxychloroquine-induced toxic myopathy causing respiratory failure. Chest $2007 ; 131: 588-90$.

10. Ratliff NB, Estes ML, Myles JL, Shirey EK, McMahon JT. Diagnosis of chloroquine cardiomyopathy by endomyocardial biopsy. N Engl J Med 1987; 316 : 191-3.

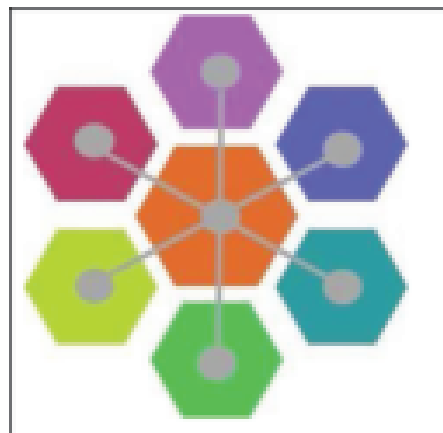

\section{Global Registry for COL6-related dystrophies}

\section{Registre global des dystrophies liées au collagène de type VI}

\section{S'Inscrire sur : www.collagen6.org}

\section{Ou contactez-nous par e-mail à radresse : collagenbregistryancl.ac.uk}

La traduction française sera bientôt disponible sur le site web.
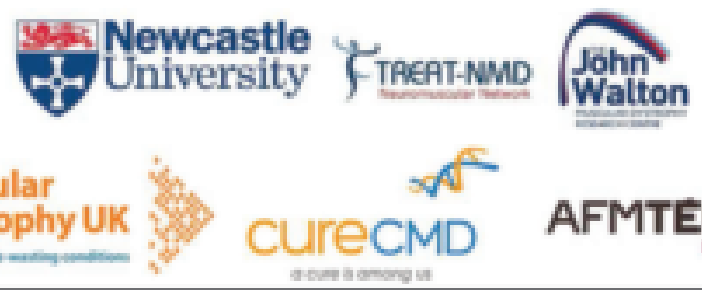